\section{Lösungskalorimetrische Bestimmung der Bildungswärmen von festen Blei-Zinn-Legierungen}

\author{
Von Franz-Josef Gilhaus \\ Max-Planck-Institut für Eisenforschung, Düsseldorf \\ (Z. Naturforschg. 14 a, 1001-1002 [1959]; eingeg. am 21. August 1959)
}

Lösungskalorimetrische Verfahren ${ }^{1-4}$, die in den letzten Jahren mehrfach angewendet wurden, dienen zur Messung von Bildungswärmen der Metall-Legierungen. Die Legierungen werden in einer Metall-Schmelze aufgelöst und die dabei auftretenden Wärmetönungen gemessen. Ein Mol der Legierung $\mathrm{AB}$ bestehe aus $x$ Teilen der Komponente A und entsprechend $(1-x)$ Teilen der Komponente B. Die Legierung habe dieselbe Temperatur wie die Lösungs-Schmelze. Wird nun die Wärmetönung $H(x)$ beim Auflösen in der Schmelze gemessen, so gilt für die Bildungswärme $\Delta H(x)$ der festen Legierung:

$$
\Delta H(x)=x \cdot H_{\mathrm{A}}+(1-x) H_{\mathrm{B}}-H(x) .
$$

Hierin bedeuten $H_{\mathrm{A}}$ und $H_{\mathrm{B}}$ die Wärmetönungen beim Auflösen je eines Mols der reinen Komponenten. Als Lösungsmittel kann die flüssige Phase eines der beiden Legierungselemente, ein anderes Metall oder eine Metall-Legierung verwendet werden

Die vorliegenden Messungen wurden mit bleireichen Blei-Zinn-Legierungen durchgeführt. Als Lösungsschmelze wurde flüssiges Zinn bei $240{ }^{\circ} \mathrm{C}$ verwendet. Bei dieser Temperatur bestehen feste bleireiche $\alpha$-Mischkristalle bis etwa über $20 \mathrm{At} . \%$ Zinn. Das System Blei-Zinn wurde hauptsächlich deshalb gewählt, weil Messungen bei verhältnismäßig niedriger Temperatur möglich sind. Die Erfahrungen aus diesen Untersuchungen sollen Messungen bei höheren Temperaturen zugute kommen.

Das verwendete Kalorimeter besteht aus einem Thermostaten, der mit (auf $\pm 2 \%$ ) geregelter Spannung geheizt wird, und einer im Thermostaten befindlichen Zwillingsanordnung zur Messung der Wärmetönungen. Diese besteht aus zwei thermisch symmetrisch angeordneten Gefäßen (d.h. die Wärmewiderstände von den Gefäßen zu dem Thermostaten sind gleich), die gleiche Mengen (je $75 \mathrm{~g}$ ) flüssiges Zinn enthalten. Eine Thermosäule mit zweimal 42 Lötstellen mißt den Temperaturunterschied zwischen den beiden Gefäßen. Dieser wird über einen Mikrovoltverstärker mit einem Schnellschreiber registriert.

Die Legierungen wurden sorgfältig unter Vakuum ( $\sim 10^{-3}$ Torr, verdünnte Argon-Atmosphäre) erschmolzen und $1 \mathrm{~h}$ bei $500{ }^{\circ} \mathrm{C}$ geglüht, in Öl von Raumtemperatur abgeschreckt und dann mehrere Tage bei $190^{\circ} \mathrm{C}$ unter Leuchtgasatmosphäre homogenisiert. Die Abweichungen des Zinngehaltes vom Sollwert betrugen weniger als $\pm 0,5 \%$ von der Konzentration.

1 O. J. Kleppa, J. Phys. Chem. 59, 175 [1955].

2 F. Wever u. B. Ilschner, Forschungsber. d. Wirtsch.- u. Verk.-Min. NRW Nr. 460 [1957].
Bei der Vorbereitung der Proben (etwa $100 \mathrm{mg}$ schwer) vor dem Einbringen ins Kalorimeter war eine Abkühlung unvermeidlich. Um die dabei auftretende Ausscheidung des Zinns aus den bleireichen Mischkristallen möglichst gering zu halten, wurden die Proben in Alkohol von Null Grad abgeschreckt, schnell gewaschen, am Einwurfmechanismus befestigt und auf Kalorimeter-Temperatur erwärmt. Dann wurden sie 20-30 min kurz oberhalb der Zinnschmelze gehalten, so daß sie deren Temperatur annehmen und daß evtl. erfolgte Ausscheidungen wieder aufgelöst werden konnten.

Nach dieser Vorbehandlung wurden die Proben zur Messung in die Zinnschmelze eingeworfen. Über diese wurde Argon als Schutzgas geleitet, um die Oxydation herabzusetzen. Sobald eine Probe in der Zinnschmelze aufgelöst wird, entsteht an der Thermosäule eine zeitlich veränderliche Spannung $s(t)$, die zunächst (absolut) zunimmt, durch einen Extremwert geht und dann exponentiell in z. B. 20 min auf den Anfangswert abfällt. Die in der Zeiteinheit abgegebene (aufgenommene) Wärmemenge $\dot{Q}(t)$ hängt mit $s(t)$ zusammen durch die Gleichung

$$
\dot{Q}(t)=a \cdot s(t)+b \cdot \dot{s}(t)
$$

mit den Konstanten $a$ und $b$. Unter Beachtung von $s(0)=s(\infty)=0$ erhält man aus (2) durch Integration die gesamte beim Lösungsvorgang abgegebene (aufgenommene) Wärmemenge $Q$ :

$$
Q=a \cdot \int_{t=0}^{\infty} s(t) \mathrm{d} t .
$$

Das rechtsstehende Integral wurde graphisch aus den gemessenen Werten ermittelt. Die Konstante $a$ wurde durch eine Eichung mit Hilfe des Peltier-Effektes bestimmt. Diese erwies sich im vorliegenden Fall genauer als eine Eichung mit dem Joule-Effekt. Die Genauigkeit und Reproduzierbarkeit der Strommessung bei der Eichung beträgt etwa $\pm 0,2$ bis $\pm 0,5 \%$, die der zur Eichung auch erforderlichen Temperaturmessung etwa $\pm 3 \%$, so daß der Fehler in der Bestimmung der Konstanten $a 1 \%$ nicht überschreitet.

In Tab. 1 sind die mit den bisherig untersuchten Legierungen gewonnenen Ergebnisse aufgeführt. Die Wärmetönungen $H(x)$ wurden aus 10 bis 12 Einzelmessungen ermittelt.

\begin{tabular}{|c|c|c|c|c|}
\hline $\begin{array}{c}\text { Gew.-\% } \\
\text { Sn }\end{array}$ & $\begin{array}{c}\text { Atom-\% } \\
\text { Sn }\end{array}$ & $\begin{array}{c}\text { Temp. } \\
{ }^{\circ} \mathrm{C}\end{array}$ & $\begin{array}{c}\text { ges. Wärme- } \\
\text { tönung } H(x) \\
{[\mathrm{cal} / \mathrm{mol}]}\end{array}$ & $\begin{array}{c}\text { mittlerer } \\
\text { Fehler } \\
{[\mathrm{cal} / \mathrm{mol}]}\end{array}$ \\
\hline 2 & 3,44 & 241 & 2277 & \pm 18 \\
4 & 6,78 & 242 & 2154 & \pm 20 \\
10,7 & 17,30 & 239,5 & 1894 & \pm 14 \\
0 & 0 & 241,5 & 2428 & \pm 5 \\
\hline
\end{tabular}

Tab. 1.

3 R. A. Oriani u. W. K. Murphy, J. Phys. Chem. 62, 327 [1958].

4 W. K. Murphy u. R. A. Oriani, Acta Met. 6, 556 [1958]. 
Mit diesen Werten wurden die Bildungswärmen $\Delta H(x)$ nach (1) bestimmt. Für die Lösungswärme $H_{\Lambda}$ des reinen Zinns wurde seine Schmelzwärme von $1720 \mathrm{cal} / \mathrm{g}$-At. genommen ${ }^{5}$. Die Lösungswärme $H_{\mathrm{B}}$ $=H(0)$ von Blei in Zinn wurde gemessen (s. Tab. 1).

Abb. 1 zeigt die nach (1) aus den kalorimetrischen Messungen bestimmten Bildungswärmen in Abhängigkeit von der Zinn-Konzentration. Zum Vergleich sind Messungen von Murphy und Oriani ${ }^{4}$ sowie die theoretischen Berechnungen von Borelius, Larris und OHLSson $^{6}$ mit eingezeichnet. Weiter ist noch ein Meßwert eingetragen, der vom Verfasser aus Untersuchungen mit einem Ausscheidungs-Kalorimeter ${ }^{7}$ gewonnen wurde. Unter Berücksichtigung der Fehlergrenzen liegen sämtliche Meßpunkte auf einer Kurve. Der ziemlich große Fehler der Ausscheidungsmessung ist durch die Unsicherheit des Phasen-Diagramms bedingt. Dagegen ist die Abweichung der von Borelius aus dem Verlauf der Phasengrenze errechneten Werte beträchtlich.

Diese Diskrepanz wird noch deutlicher, wenn aus den gemessenen Bildungswärmen unter Annahme idealer Mischungsentropie der Verlauf der freien Energie für die bleireichen Mischkristalle berechnet wird. Nach dieser Rechnung wäre Zinn in Blei fast unlöslich.

Die Abweichung der gemessenen Bildungswärmen von den aus dem Phasen-Diagramm berechneten liegt darin begründet, daß die Lösung des Zinns in festem

5 Recueil de Données Thermodynamiques, Hrsg. IRSID, Metz 1955.

${ }^{6}$ G. Borelius, F. Larris u. E. Ohlssen, Ark. Met. Astr. Fys. 31 A, No. 10 [1944].

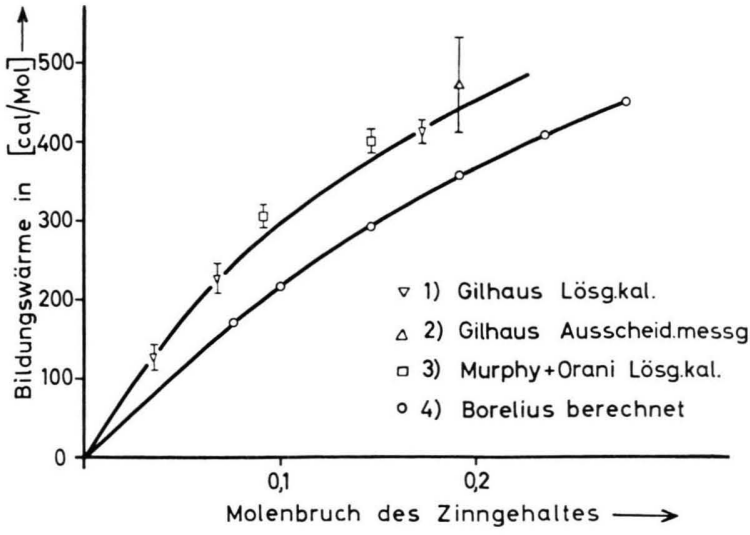

Abb. 1. Bildungswärmen $\Delta H$ im System Blei-Zinn als Funktion der Konzentration.

Blei in einem kubisch flächenzentrierten Gitter erfolgt, während festes Zinn ein anderes Kristallgitter hat. Für die Größe $H_{\mathrm{A}}$ in (1) wäre die Schmelzwärme eines (hypothetischen) kubisch flächenzentrierten Zinns einzusetzen, um Úbereinstimmung mit dem Phasen-Diagramm zu erzielen. Ähnliche Verhältnisse können in anderen Legierungs-Systemen vorliegen.

7 F. Wever, O. Krisement u. H. Schädler, Forschungsber. d. Wirtsch.- u. Verk.-Min. NRW, Nr. 459 [1957].

\section{Zur Messung der Temperaturabhängigkeit der Lumineszenz von CdS}

Von Klaus Albers

II. Physikalisches Institut der Humboldt-Universität zu Berlin (Z. Naturforschg. 14 a, 1002-1003 [1959] ; eingeg. am 14. September 1959)

Untersuchungen der Temperaturabhängigkeit der Lumineszenz von CdS ergeben bekanntlich bei Zimmertemperatur rote und infrarote Lumineszenz, welche sich nach Messungen von Boldt, Link und Seiwert ${ }^{1,2}$ mit zunehmender Temperatur je nach Art des Kristalles in der Intensität ändert. Bei Erwärmung über $50^{\circ} \mathrm{C}$ zeigen jedoch alle Kristalle übereinstimmend eine deutliche Abnahme der Intensität der roten Bande, und zwischen $100^{\circ}$ und $200{ }^{\circ} \mathrm{C}$ ist diese Lumineszenz bei allen untersuchten Kristallen fast vollständig verschwunden. Die genannten Autoren führen diese Temperaturabhängigkeit z. Tl. auf Umwandlungen der Lumineszenz-Zentren zurück, da die Lumineszenzfähigkeit der einmal so hoch erwärmten Kristalle auch nach Abkühlung auf Zimmertemperatur sehr viel geringer als im Anfangszustand ist. Bei manchen Kristallen scheint sie fast vollständig verlorengegangen zu sein.

1 R. Link u. R. Seiwert, Z. phys. Chemie, im Erscheinen.

2 W. Boldt u. R. Seiwert, Z. phys. Chemie, im Erscheinen.
In Fortführung der Arbeiten der genannten Autoren haben wir kürzlich gefunden, daß die gelöschten Kristalle durch geeignete thermische Behandlung ihre Lumineszenzfähigkeit wiedererlangen. Erwärmt man nämlich diese Kristalle in einem Vakuum von $10^{-4}$ bis $10^{-5}$ Torr bei Dunkelheit auf etwa $150^{\circ}$ bis $200^{\circ} \mathrm{C}$ und läßt sie dann wieder abkühlen, so erreicht die Lumineszenz bei gleicher Anregung nahezu dieselbe Intensität wie vor der Behandlung. Die Messungen, die sowohl mit $\lambda=365 \mathrm{~m} \mu$ als auch mit $\lambda=435 \mathrm{~m} \mu$ als anregender Wellenlänge an verschiedenen Kristallen durchgeführt wurden, zeigten stets dasselbe Ergebnis. Dabei kann man bei ein und demselben Kristall die Lumineszenz anscheinend beliebig oft nacheinander verschwinden lassen und wieder hervorrufen.

Nach unseren bisherigen Untersuchungen handelt es sich bei der Löschung der roten Lumineszenz weder um einen reinen Temperatureffekt noch um eine irreversible thermische Strukturumwandlung, sondern um einen Prozeß, der durch Zusammenwirken von thermischer Energie und kurzwelligem Licht eine Umwandlung von Lumineszenz-Zentren bewirkt, die aber durch thermische Energie ohne Einstrahlung rückgängig gemacht werden kann. Denn daß das anregende Licht bei dem Prozeß der Löschung eine wesentliche Rolle spielt, zeigt unsere Beobachtung, nach der die in Dunkelheit auf 150 bis 